
ММР-2, ММР-3 В РАЗВИТИИ АНТРАЦИКЛИН-ИНДУЦИРОВАННОЙ КАРДИОМИОПАТИИ И. А. Карпуть ${ }^{1}$ В. А. Снежицкий ${ }^{1}$ М. Н. Курбат ${ }^{1}$, А. Ю. Рубинский ${ }^{2}$ Т. А. Смирнова ${ }^{1}$ Гродненский государственный медииинский университет, Гродно, Беларусь ${ }^{2}$ Гродненский областной клинический кардиологический центр, Гродно, Беларусь ${ }^{3}$ Гродненская университетская клиника, Гродно, Беларусь

В обзоре рассматривается актуальная проблема кардиотоксичности химиопрепаратов. Противоопухолевые антибиотики антрациклины способны вызывать кардиомиопатию, которая может ухудшать прогноз у онкопациентов и быть самостоятельной причиной смерти.

Выявление пациентов с высоким риском кардиотоксичности - первый шаг на пути к успешной профилактике сердечной недостаточности у онкобольных. Генетическое типирование может стать эффективной мерой прогнозирования повышенного риска кардиотоксического действия антрациклинов.

В обзоре проанализирована роль мутаций генов при развитии сердечной недостаточности, индуцированной химиотерапией.

Ключевые слова: химиотерапия, кардиотоксичность, белок титин, матриксные металлопротеиназы.

Для цитирования: Роль полиморфизмов генов TTN, TTN-truncation, MMP-2, MMP-3 в развитии антрациклин-индуциированной кардиомиопатии / И. А. Карпуть, В. А. Снежиикий, М. Н. Курбат, А. Ю. Рубинский, Т. А. Смирнова // Журнал Гродненского государственного медищинского университета. 2021. T. 19, № 2. C. 135-140. https://doi.org/10.25298/22218785-2021-19-2-135-140.

Рак молочной железы (РМЖ) - наиболее распространенное злокачественное новообразование у женщин всего мира, включая Республику Беларусь, стабильно удерживает лидирующие позиции среди причин смертности от онкологических заболеваний [1].

Совершенствование методов ранней диагностики и значительный прогресс противоопухолевой терапии способствовали увеличению безрецидивной выживаемости и продолжительности жизни пациентов с РМЖ. Противоопухолевая химиотерапия (ХT) наряду с хирургической и лучевой терапией - важный компонент лечения РМЖ. ХТ включает ряд препаратов, подавляющих рост опухоли. Однако, помимо лечебного эффекта, эти препараты оказывают и токсическое действие. Таковыми являются антрациклиновые антибиотики (чаще доксорубицин), которые используются для первой линии полихимиотерапии (ПХТ) РМЖ, что обусловлено их достаточной эффективностью и экономической целесообразностью применения в клинической практике [2]. Терапевтическая эффективность антрациклинов сопряжена с развитием разного рода осложнений, среди которых наиболее распространенное - кардиотоксичность (КТ). КТ - это один из наиболее серьезных побочных эффектов противоопухолевой терапии, который ведет к развитию и прогрессированию сердечно-сосудистых заболеваний, в ряде случаев с фатальным исходом. Так, смертность пациентов, у которых развилась сердечная недостаточность $(\mathrm{CH})$ на фоне лечения рака, достигает $60 \%$ в течение 2 лет [3]. КТ остается особо важной проблемой, учитывая большое число женщин с РМЖ, получавших терапию антрациклинами. Сердечно-сосудистые осложнения у женщин, получавших терапию антрациклинами, возникают в 4-36\% случаев. В среднем $17 \%$ пациентов, получающих такое лечение при наиболее агрессивных формах РМЖ, вынуждены прекратить лечение из-за сердечно-сосудистых заболеваний [1].

Несмотря на достижение определенной успешности в управлении риском КТ, вызванной химиопрепаратами в процессе активного лечения, стало очевидным, что нередко могут возникать и ее отсроченные проявления. В связи с этим более актуальная задача исследователей в настоящее время - поиск методов, с помощью которых можно было бы оценить индивидуальный риск развития заболевания. Накопленные данные о механизмах развития антрациклинопосредованной кардиотоксичности (АОК) свидетельствуют о большом значении ряда генов, определяющих риск развития АОК в ходе химиотерапии онкологических заболеваний. Таким образом, альтернативный подход - использование генетического типирования для прогнозирования риска развития КТ на фоне лечения РМЖ [4]. Информация о возможном высоком индивидуальном риске кардиотоксического действия антрациклинов, полученная при генотипировании, позволит онкологам корректировать химиотерапевтические режимы еще до начала лечения опухолевого заболевания и/или позволит кардиологам провести профилактику СН [5].

\section{Связь генов TTN и TTN-truncation с антрациклин-опосредованной кардиотоксичностью}

В настоящее время общепризнан факт несомненной роли молекулярно-генетических факторов в развитии кардиомиопатии в результате кардиотоксического действия ХT [4]. Антрациклиновая КТ на фоне лечения РМЖ сопровождается развитием дилатационной кардиомиопатии (ДКМП) и в конечном итоге - развитием застойной СН. В последнее десятилетие накопились многочисленные данные, свидетельствующие о том, что заболеваемость ДКМП посте- 
пенно и неуклонно прогрессирует и радикально отличается своей крайней гено- и фенотипической гетерогенностью. Перекрывание негенетических факторов с фенотипом генетических расстройств приводит к более неоднозначным клиническим проявлениям ДКМП и повышает сложность диагностики данного заболевания [6].

С развитием методов секвенирования нового поколения (new generation sequencing, NGS) стали проводиться по-настоящему масштабные генетические исследования ДКМП. В результате этих исследований впервые был определен ген, мутации в котором встречались у значительной доли лиц с ДКМП - ген ТTN, кодирующий саркомерный белок титин. Ген TTN - самый большой из известных генов в геноме человека. По разным оценкам, от 10 до 30\% случаев ДКМП обусловлены мутациями в гене TTN [7].

Белок титин определяет пассивное сопротивление саркомеров при растяжении [6]. Как известно, титин экспрессируется в двух формах - N2B и N2BA. B миокарде преобладает менее упругая форма 2BA и соотношение N2BA/N2B - положительное. Превалирование формы N2BA объясняется необходимостью обеспечить растяжение саркомеров миокарда [8]. Роль доксорубицина в развитии кардиотоксичности была представлена в экспериментальной модели на белых кроликах. Исследование показало, что при введении доксорубицина отмечается тенденция к повышению активного напряжения кардиомиоцитов, смещению в сторону менее упругой изоформы N2BA, гипофосфорилирование N2B [9].

Патогенными принято считать укорачивающие титин варианты - нонсенс и сплайсинг мутации, а также варианты, приводящие к сдвигу рамки считывания - TTN-укорачивающие/усекающие варианты (TTN-truncation). Около 15-20\% пациентов с ДКМП имеют TTN-truncation варианты. Важно отметить, что TTN-truncation варианты ассоциированы с высоким риском желудочковых аритмий, с развитием интерстициального фиброза и выраженными изменениями митохондриальной функции кардиомиоцитов [6].

Современные исследования предполагают, что пациенты с кардиомиопатией, индуцированной при лечении рака, имеют избыток редких мутаций в генах, отвечающих за развитие ДКМП, и наиболее часто встречаемый среди них - ген TTN. Исследования показали, что 7,5\% из 213 пациентов с кардиомиопатией, индуцированной при лечении рака, имели варианты усечения в TTN гене. K тому же у пациентов с кардиомиопатией и TTN-усекающими вариантами (TTNtv TTN truncating variants) диагностировано больше случаев СН, фибрилляции предсердий, дисфункции миокарда, в отличие от пациентов без TTNtv. В дополнение к TTNtv в ходе исследования выявлены редкие белок-изменяющие варианты в пяти генах ДКМП: ВАG3, LMNA, MYH7, TNNT2, TCAP. Таким образом, варианты мутаций, которые были обнаружены у 12,2\% пациентов с кардиомиопатией, индуцированной при лечении рака, встречаются примерно у 40\% пациентов с семейной и спорадической ДКМП [10].
Исследователи отмечают, что у 10\% пациентов, которые получали антрациклиновые антибиотики, выявляются случаи кардиотоксичности уже при кумулятивной дозе антрациклинов - 250 мг/м кв. Авторы сообщают, что комбинация антрациклинов с другими химиотерапевтическими препаратами, такими как трастузумаб, приводит к снижению фракции выброса левого желудочка у 34\% пациентов, а клинические проявления СН наблюдаются у 2-4\% пациентов. А также другие факторы риска - женский пол, возраст, сопутствующая сердечная патология и т. д. - также провоцируют развитие кардиомиопатии [11]. Однако при учете всех этих факторов предикторы индивидуальной предрасположенности остаются сложной задачей. Ученые отметили, что TTN-усекающие варианты при ДКМП выявляются у $25 \%$ пациентов с терминальной стадией заболевания и у $15 \%$ - в субклинической стадии заболевания. При кардиомиопатии, индуцированной лечением рака, у 8,1\% пациентов были выявлены эти опасные TTN-усекающие варианты [10].

Проведено также секвенирование поколений в трех когортах пациентов с индуцированной кардиотоксичностью. Когорты включали пациентов, страдающих раком молочной железы, с гематологической злокачественной патологией и другими солидными злокачественными опухолями. Все пациенты получали антрациклиновые антибиотики с суммарной дозой 400 мг/м кв. Дополнительные исследования выполнены на 40 других генах, участвующих в развитии кардиомиопатии, но там не было выявлено значительных различий в распространенности редких белок-изменяющих вариантов или вариантов как предикторов повреждения [12].

Роль TTN-усекающих вариантов в развитии антрациклиновой кардиотоксичности была подтверждена на экспериментальной модели. Гетерозиготные мыши с TTN-усекающими вариантами и «wildtype» получали три дозы доксорубицина с недельным интервалом (кумулятивная доза 45 мг/м кв). Функция левого желудочка была схожей у мышей с TTN-усекающими вариантами на исходном уровне и без таковых, причем сравнительно одинаково снижалась после 4 недель применения антрациклиновых антибиотиков. Функция левого желудочка была восстановлена к восьмой неделе у мышей «wildtype», но оставалась сниженной через 12 недель у мышей с TTN-усекающими вариантами [10].

Таким образом, наличие и увеличение в геноме количества редких вариантов, включающих TTNtv, дает возможность предположить, что данные генетические изменения могут быть предиктором повышенного риска развития АОК. Эти данные устанавливают факт генетической связи между ДКМП и кардиомиопатией, индуцированной антрациклиновыми антибиотиками.

\section{Роль матриксных металлопротеиназ \\ в развитии антрациклин-индуцированной кардиотоксичности}

Молекулярные механизмы антрациклин-зависимого ремоделирования миокарда до конца 
не выяснены. В частности, большое значение имеет исследование механизмов протеолиза структурных белков кардиомиоцитов, обуславливающих развитие $\mathrm{CH}$.

Развивающиеся дисфункции миокарда часто ассоциируются с изменениями активности некоторых матриксных металлопротеиназ, обнаруживаемых как во внеклеточном матриксе миокарда, так и в кардиомиоцитах. Доказано, что металлопротеиназа-2 (ММР-2) экспрессируется в кардиомиоцитах в процессе их пролиферации; в саркомерах миофибриллярных пучков и в ядрах кардиомиоцитов при развитии окислительного стресса. Таким образом, обнаружение внутриклеточных локализаций ММР-2 и установление интенсивности ее экспрессии в миокарде при разных патологических и физиологических состояниях могут быть использованы в изучении молекулярно-клеточных основ развития сердечной дисфункции [13]. Недавние исследования показали, что нарушение структурной целостности титина напрямую связано с развитием ДКМП. Известно, что доксорубицин вызывает быструю деградацию титина через активацию протеолитических путей [8]. При этом доксорубицин, усиливая окислительный стресс с образованием свободных радикалов, активных форм кислорода, активных метаболитов путем перекисного окисления липидов, стимулирует ММР-2, которая вызывает протеолиз титина.

Роль ММР-2 в развитии антрациклиновой кардиотоксичности была исследована на экспериментальной модели. В результате исследования установлено, что доксорубицин увеличивает активность миокардиального МMP-2 в сердце мышей и в кардиомиоцитах, полученных из стволовых эмбриональных клеток человека, частично за счет denovo экспрессии N-концевой усеченной ММР-2 изоформы, которая экспрессируется посредством окислительного стресса. Повышение уровня ММР-2 в сердце при антрациклиновой кардиотоксичности способствует ремоделированию левого желудочка, интерстициальному фиброзу и протеолизу титина [13].

Рядом исследователей предложен способ прогнозирования развития антрациклин-индуцированной кардиотоксичности с использованием генетических маркеров риска, в основе которого лежит участие матриксных металлопротеиназ в ремоделировании сердца и сосудов. Так, матриксная металлопротеиназа-3 (ММР-3) играет значимую роль в естественных процесcax ремоделирования тканей и в патологиче-

\section{Литература}

1. Оценка субклинической кардиотоксичности антрациклинсодержащей химиотерапии рака молочной железы в зависимости от кумулятивной дозы доксорубицина и исходного уровня артериального давления / А. А. Авалян [и др.] // Системные гипертензии. - 2018. - Т. 15, № 4. - С. 59-64. - doi:10.2 6442/2075082X.2018.4.000021.

2. Поиск ранних маркеров кардиотоксичности противоопухолевого лечения у больных раком ских процессах, при этом полиморфный вариант 5A/6А гена ММР-3 играет важную роль в регулировании уровня ММР-3 и представляет собой оптимальный вариант генотипа МMP-3. Для прогнозирования развития антрациклин-индуцированной кардиотоксичности определен полиморфизм гена ММР-3 (полиморфного локуса $5 \mathrm{~A} / 6 \mathrm{~A}$ (rs3025058)). Высокий риск развития КТ прогнозируют у носителей генотипа 5A/5A полиморфного локуса 5A/6А гена ММР-3 [14].

Таким образом, опираясь на результаты исследования генотипа матриксной металлопротеиназы-3, которая участвует в ремоделировании сердца при применении антрациклиновых антибиотиков, было бы целесообразно изучить активность ММР-2 и ММР-3 при антрациклин-опосредованной кардиомиопатии, а также исследовать генотип ММР-2 с целью поиска возможных генетических маркеров риска антрациклин-индуцированной кардиотоксичности.

\section{Заключение}

Антрациклин-опосредованная кардиотоксичность - важная проблема современной медицины, пока не имеющая четкого плана ее решения. Существует несколько стратегий для уменьшения риска АОК, хотя ни одна из них не характеризуется надежными подтверждениями, полученными в контролируемых клинических исследованиях или стандартизированной клинической практике. Несмотря на достижение определенных успехов в разработке методов ранней диагностики заболевания, необходимы дальнейшие исследования для изучения диагностической точности и воспроизводимости показателей этих методов, а также их стандартизация.

Результаты многих исследований свидетельствуют о необходимости персонифицированного подхода к оценке факторов риска развития КТ у пациентов с РМЖ на этапе подготовки к проведению полихимиотерапии антрациклинами. На наш взгляд, изучение полиморфизма генов, ассоциированных с антрациклиновой кардиотоксичностью, может применяться для выявления повышенного риска развития $\mathrm{CH}$ у пациентов с РМЖ до проведения ХТ. Другими словами, информация о возможном высоком индивидуальном риске кардиотоксического действия антрациклинов, полученная при генотипировании, позволит при междисциплинарном взаимодействии кардиологов и онкологов выработать новую стратегию преодоления этой проблемы еще до начала лечения опухолевого заболевания.

молочной железы в зависимости от уровня артериального давления / А. А. Авалян [и др.] // Системные гипертензии. - 2017. - Т. 14, № 3. - С. 2127. - doi:10.26442/2075-082X 14.3.21-27.

3. Возможности современных эхокардиографических технологий в ранней диагностике кардиотоксического действия химиотерапевтических препаратов антрациклинового ряда у онкологических больных / Ю. А. Васюк [и др.] // Кардиология. - 2017. - Т. 57, № 4S. - C. 31-37. - doi: 10.18087/cardio. 2417. 
4. Роль полиморфных вариантов генов NO-синтазы, peцептора эндотелина-1 и NADPH-оксидазы при развитии сердечной недостаточности, индуцированной кардиотоксичностью химиотерапии / А. Т. Тепляков [и др.] // Комплексные проблемы сердечно-сосудистых заболеваний. - 2018. - Т. 7, № 3. - С. 33-43. - doi: 10.17802/2306-1278-2018-7-3-33-43.

5. Состояние сердечно-сосудистой системы у больных с антрациклиновой кардиомиопатией / А. Т. Тепляков [и др.] // Бюллетень сибирской медицины. - 2017. - Т. 16, № 3. - C. 127-136. - doi: 10.20538/1682-0363-2017-3127-136.

6. Дилатационная кардиомиопатия: новый взгляд на проблему / Т. Г. Вайханская [и др.] // Российский кардиологический журнал. - 2019. - Т. 24, № 4. - С. 35-47. - doi: 10.15829/1560-4071-2019-4-35-47.

7. Молекулярно-генетический анализ гена TTN у детей с дилатационной кардиомиопатией / В. С. Михайлов [и др.] // Клиническая и экспериментальная хирургия. Журнал имени академика Б.В. Петровского. - 2018. - Т. 6, № 1. - C. 70-76. - doi: 10.24411/2308-1198-2018-00010.

8. Современные представления о методах диагностики и профилактики антрациклин-опосредованной кардиотоксичности у больных раком молочной железы [Электронный ресурс] / Л. Н. Ващенко [и др.] // Современные проблемы науки и образования. -2019. - № 6. - Режим доступа: http://science-education.ru/ru/ article/view?id=29347. - Дата доступа: 10.10.2020.

9. Early myocardial changes induced by doxorubicin in the nonfailing dilated ventricle / P. G. Rodrigues [et al.] // American Journal of Physiology: Heart and Circulatory Physiology. - 2019. - Vol. 316, № 3. - P. H459-H475. doi: 10.1152/ajpheart.00401.2018.

10. Genetic variants associated with cancer therapy-induced cardiomyopathy / P. Garcia-Pavia [et al.] // Circulation. - 2019. - Vol. 140, № 1. - P. 31-41. - doi: 10.1161/ CIRCULATIONAHA.118.037934.

11. Леонтьева, И. В. Дифференциальная диагностика гипертрофической кардиомиопатии / И. В. Леонтьева // Российский вестник перинатологии и педиатрии. 2017. - T. 62, № 3. - C. 20-31. - doi: 10.21508/10274065-2017-62-3-20-31.

12. Genomics - First Evaluation of Heart Disease Associated with Titin-Truncating Variants / C. M. Haggerty [et al.] // Circulation. - 2019. - Vol. 140, № 1. - P. 42-54. - doi: 10.1161/ CIRCULATIONAHA.119.039573.

13. MMP inhibitors attenuate doxorubicin cardiotoxicity by preventing intracellular and extracellular matrix remodeling / B. Y. H. Chan [et al.] // Cardiovascular Research. - 2020. Vol. 116, № 2. - P. 1238-1245. - doi: 10.1093/cvr/cvaa017.

14. Способ прогнозирования антрациклин-индуцированной кардиотоксичности у женщин с раком молочной железы : патент RU 2714683C1 / А. T. Тепляков, Е. B. Гракова, С. Н. Шилов, Е. Н. Березикова, А. А. Попова, М. Н. Неупокоева, К. В. Копьева ; Томский национальный исследовательский медицинский центр Российской академии наук. - Опубл. 19.02.20.

15. Оценка роли полиморфизма гена матриксной металлопротеиназы-3 в развитии хронической сердечной недостаточности / А. Т. Тепляков [и др.] // Терапевтический архив. - 2015. - Т. 87, № 4. - С. 8-12. - doi: 10.17116/terarkh20158748-12.

16. Прогностическое значение биомаркеров предшественника мозгового натрийуретического пептида и растворимого Fas-лигада в оценке риска кардиотоксичности антрациклиновой химиотерапии / А. Т. Тепляков [и др.] // Кардиоваскулярная терапия и профилактика. - 2019. - Т. 18, № 1. - С. 127-133. - doi: 10.15829/1728-8800-2019-1-127-133.

17. Сердечно-сосудистые осложнения после противоопухолевой терапии у онкологических больных в отдаленные сроки ( $>1$ года) / М. Л. Казанцева [и др.] // Евразийский кардиологический журнал. - 2019. - № 3. - С. 50-57.

18. Трушин, А. Ю. Трастузумаб-индуцированная кардиотоксичность - $\quad$ м междисциплинарная проблема современной медицины / А. Ю. Трушин // Международный научно-исследовательский журнал. - 2019. - T. 82, № 4-1. - C. 118-123. - doi: 10.23670/ IRJ.2019.82.4.024.

19. Disruption of a GATA4/Ankrd1 signaling axis in cardiomyocytes leads to sarcomere disarray: implications for anthracycline cardiomyopathy / B. Chen [et al.] // PLoS One. - 2012. - Vol. 7, № 4. - Art. e3574. - doi: 10.1371/ journal.pone.0035743.

20. Doxorubicin induces de novo expression of N-terminaltruncated matrix metalloproteinase-2 in cardiac myocytes / B. Y. H. Chan [et al.] // Canadian Journal of Physiology and Pharmacology. - 2018. - Vol. 96, № 12. - P. 12381245. - doi: 10.1139/cjpp-2018-0275.

21. Genetic epidemiology of titin-truncating variants in the etiology of dilated cardiomyopathy / A. M. Tabish [et al.] // Biophysical Reviews. - 2017. - Vol. 9, № 3. - P. 207-223. - doi: 10.1007/s12551-017-0265-7.

22. Henriksen, P. A. Anthracycline cardiotoxicity: an update on mechanisms, monitoring and prevention / P. A. Henriksen // Heart. - 2018. - Vol. 104, № 12. P. 971-977. - doi: 10.1136/heartjnl-2017-3121103.

23. Matrix metalloproteinase- 2 in oncostatin M-induced sarcomere degeneration in cardiomyocytes / X. Fan [et al.] // American Journal of Physiology: Heart and Circulatory Physiology. - 2016. - Vol. 311, № 1. - P. H183-H189. doi: 10.1152/ajpheart.00229.2016.

24. Prevention and Monitoring of Cardiac Dysfunction in Survivors of Adult Cancer: American Society of Clinical Oncology Clinical Practice Guideline / S. H. Armenian [et al.] // Journal of Clinical Oncology. - 2017. - Vol. 35, № 8. - P. 893-911. - doi: 10.1200/JCO.2016.70.5400.

25. Titin-truncating variants are associated with heart failure events in patients with left ventricular non-compaction cardiomyopathy / S. Li [et al.] // Clinical Cardiology. - 2019. - Vol. 42, № 5. - P. 530-535. - doi: 10.1002/clc.23172.

26. Titin Truncating Variants in Dilated cardiomyopathy - Pprevalence and Genotype-Phenotype Correlations / M. Franaszczyk [et al.] // PLoS One. - 2017. - Vol. 12, № 1. - Art. e0169007. - doi: 10.1371/journal.pone.0169007.

27. Truncating Titin (TTN) Variants in Chemotherapyinduced Cardiomyopathy / M. Linschoten [et al.] // Journal of Cardiac Failure. - 2017. - Vol. 23, № 6. - P. 476-479. - doi: 10.1016/j.cardfail.2017.03.003.

\section{References}

1. Avalyan AA, Oshhepkova EV, Saidova MA, Shitov VN, Glazkova EV, Stenina MB, Chazova IE. Ocenka subklinicheskoj kardiotoksichnosti antraciklinsoderzhashhej himioterapii raka molochnoj zhelezy v zavisimosti ot kumuljativnoj dozy doksorubicina i ishodnogo urovnja arterialnogo davlenija [Evaluation of subclinical cardiotoxicity in patients with breast cancer and arterial hypertension in two regimens of anthracycline-containing chemotherapy]. Sistemnye gipertenzii [Systemic Hypertension]. 2018;15(4):59-64. doi: 10.26442/2075082H.2018.4.0000 21. (Russian).

2. Avalyan AA, Kirillova MYu, Shitov VN, Oshhepkova YeV, Saidova MA, Stenina MB, Chazova IYe. Poisk 
rannih markerov kardiotoksichnosti protivoopuholevogo lechenija u bolnyh rakom molochnoj zhelezy v zavisimosti ot urovnja arterialnogo davlenija [Markers of really cardiotoxicity in patients with breast cancer undergoing chemotherapy depending on blood pressure level]. Sistemnye gipertenzii [Systemic Hypertension]. 2017;14(3):21-27. doi: 10.26442/2075-082H_14.3.21-27. (Russian).

3. Vasyuk JuA, Nesvetov VV, Shkolnik EL, Fursov SA, Shkolnik LD, Gendlin GE, Emelina EI. Vozmozhnosti sovremennyh jehokardiograficheskih tehnologij $\mathrm{v}$ rannej diagnostike kardiotoksicheskogo dejstvija himioterapevticheskih preparatov antraciklinovogo rjada u onkologicheskih bolnyh [Possibilities of modern echocardiographic technologies in the early diagnosis of the cardiotoxic effect of chemotherapy drugs antracycline series in cancer patients]. Kardiologija. 2017;57(4):31-37. doi: 10.18087/ cardio.2417. (Russian).

4. Teplyakov AT, Shilov SN, Popova AA, Berezikova EN, Grakova EV, Neupokoeva MN, Valeeva AM, Tuleutaev ShM, Kopeva KV. Rol polimorfnyh variantov genov NOsintazy, receptora jendotelina-1 i NADPH-oksidazy pri razvitii serdechnoj nedostatochnosti, inducirovannoj kardiotoksichnostju himioterapii [The role of polymorphic variants of NO-synthase, receptor endothelin-1 and NADFHoxidase in the development of heart failure resulted from chemotherapy-induced cardiotoxicity]. Kompleksnye problemy serdechno-sosudistyh zabolevanij [Complex Issues of Cardiovascular Diseases]. 2018;7(3):33-43. doi: 10.17802/2306-1278-2018-7-3-33-43. (Russian).

5. Teplyakov AT, Shilov SN, Popova AA, Grakova EV, Berezikova EN, Neupokoeva MN, Molokov AV, Kopeva KV, Kalyuzhin VV. Sostojanie serdechno-sosudistoj sistemy u bolnyh s antraciklinovoj kardiomiopatiej [The cardiovascular system in patients with anthracycline cardiomiopathy]. Bjulleten sibirskoj mediciny [Bulletin of Siberian Medicine]. 2017;16(3):127-136. doi: 10.20538/16820363-2017-3-127-136. (Russian).

6. Vaykhanskaya TG, Sivitskaya LN, Kurushko TV, Levdansky OD, Danilenko NG. Dilatacionnaja kardiomiopatija: novyj vzgljad na problemu [Dilated cardiomyopathy: reconceptualization of the problem]. Rossijskij kardiologicheskij zhurnal [Russian Journal of Cardiology]. 2019;24(4):35-47. doi: 10.15829/1560-4071-2019-4-3547. (Russian).

7. Mikhaylov VS, Bukaeva AA, Rumyantseva VA, Kotlukova NP, Radjabova GM, Balashova MS, Dzemeshkevich SL, Zaklyazminskaya EV. Molekuljarno-geneticheskij analiz gena TTN u detej s dilatacionnoj kardiomiopatiej [Molecular genetic testing of the TTN gene in children with dilated cardiomyopathy]. Klinicheskaja i jeksperimentalnaja hirurgija. Zhurnal imeni akademika BV Petrovskogo [Clinical and experimental surgery. Petrovsky journal]. 2018;6(1):70-76. doi: 10.24411/2308-1198-2018-00010. (Russian).

8. Vashhenko LN, Ratieva AS. Gvaldin DJ, Novikova IA, Vladimirova LY, Dashkova IR, Tikhanovkaya NM, Potemkin DS. Sovremennye predstavlenija o metodah diagnostiki i profilaktiki antraciklin-oposredovannoj kardiotoksichnosti u bolnyh rakom molochnoj zhelezy [Modern concepts of diagnostic and prevention methods of antracycline-mediated cardiotoxicity in breast cancer patients]. Sovremennye problemy nauki $i$ obrazovanija [Modern problems of science and education] [Internet]. 2019;6. Available from: http://science-education.ru/ru/article/view?id=29347 (Russian).

9. Rodrigues PG, Miranda-Silva D, Costa SM, Barros C, Hamdani N, Moura C, Mendes MJ, Sousa-Mendes C, Trindade F, Fontoura D, Vitorino R, Linke WA, Leite-
Moreira AF, Falcão-Pires XI. Early myocardial changes induced by doxorubicin in the nonfailing dilated ventricle. American Journal of Physiology: Heart and Circulatory Physiology. 2019;316(3):H459-H475. doi: 10.1152/ ajpheart.00401.2018.

10. Garcia-Pavia P, Kim Y, Restrepo-Cordoba MA, Lunde IG, Wakimoto H, Smith AM, Toepfer CN, Getz K, Gorham J, Patel P, Ito K, Willcox JA, Arany Z, Li J, Owens AT, Govind R, Nuñez B, Mazaika E, Bayes-Genis A, Walsh R, Finkelman B, Lupon J, Whiffin N, Serrano I, Midwinter $\mathrm{W}$, et al. Genetic variants associated with cancer therapy-induced cardiomyopathy. Circulation. 2019;140(1):3141. doi: 10.1161/CIRCULATIONAHA.118.037934.

11. Leonteva IV. Differencialnaja diagnostika gipertroficheskoj kardiomiopatii [Differential diagnosis of hypertrophic cardiomyopathy]. Rossijskij vestnik perinatologii $i$ pediatrii [Russian Bulletin of Perinatology and Pediatrics]. 2017;62(3):20-31. doi: 10.21508/1027-4065-2017-62-320-31. (Russian).

12. Haggerty CM, Damrauer SM, Levin MG, Birtwell D, Carey DJ, Golden AM, Hatzel DN, Hu Y, Judy R, Kelly MA, Kember RL, Kirchner HL, Leader JB, Liang L, McDermott-Roe C, Babu A, Morley M, Nealy Z, Person TN, Pulenthiran A, Small A, Smelser DT, Stahl RC, Sturm AC, Williams H, et al. Genomics - First Evaluation of Heart Disease Associated with Titin-Truncating Variants. Circulation. 2019;140(1):42-54. doi: 10.1161/ CIRCULATIONAHA.119.039573.

13. Chan BYH, Roczkowsky A, Cho WJ, Poirier M, Sergi C, Keschrumrus V, Churko JM, Granzier H, Schulz R. MMP inhibitors attenuate doxorubicin cardiotoxicity by preventing intracellular and extracellular matrix remodeling. Cardiovascular Research. 2020;116(2):1238-1245. doi: 10.1093/cvr/cvaa017.

14. Teplyakov AT, Grakova EV, Shilov SN, Berezikova EN, Popova AA. Neupokoeva MN, Kopeva KV, inventors; Tomskij natsionalnyj issledovatelskij meditsinskij tsentr Rossijskoj akademii nauk, assignee. Sposob prognozirovanija antraciklin-inducirovannoj kardiotoksichnosti u zhenshhin s rakom molochnoj zhelezy [Method for prediction of anthracycline-induced cardiotoxicity in women with breast cancer]. RU patent 2714683C1. 2020 Febr 19. (Russian).

15. Teplyakov AT, Berezikova EN, Shilov SN, Grakova EV, TorimYuYu, Efremov AV, Safronov ID, Pustovetova MG, Karpov RS. Ocenka roli polimorfizma gena matriksnoj metalloproteinazy-3 v razvitii hronicheskoj serdechnoj nedostatochnosti [Assessment of the role of matrix metalloproteinase-3 gene polymorphism in the development of chronic heart failure]. Terapevticheskij arhiv [Therapeutic archive]. 2015;87(4):8-12. doi: 10.17116/ terarkh20158748-12. (Russian).

16. Teplyakov AT, Shilov SN, Popova AA, Berezikova EN, Neupokoeva MN, Grakova EV, Kopeva KV. Prognosticheskoe znachenie biomarkerov predshestvennika mozgovogo natrijureticheskogo peptida i rastvorimogo Fas-ligada v ocenke riska kardiotoksichnosti antraciklinovoj himioterapii [The prognostic value of the NT-proBNP biomarkers and Fas-ligand in assessing the risk of cardiotoxicity of anthracycline chemotherapy]. Kardiovaskuljarnaja terapija i profilaktika [Cardiovascular therapy and prevention]. 2019;18(1):127-133. doi: 10.15829/1728-88002019-1-127-133. (Russian).

17. Kazantseva ML, Oshhepkova EV, Saidova MA, Avalyan AA, Chazova IE. Serdechno-sosudistye oslozhnenija posle protivoopuholevoj terapii u onkologicheskih bolnyh v otdalennye sroki ( $>1$ goda) [Long-term cardiovascular com- 
plications in oncologic patients after antitumor therapy (>1 year)]. Evrazijskij kardiologicheskij zhurnal [Eurasian Heart Journal]. 2019;3:50-57. (Russian).

18. Trushin AY. Trastuzumab-inducirovannaja kardiotoksichnost - mezhdisciplinarnaja problema sovremennoj mediciny [Trastuzumab-induced cardiotoxicity-interdisciplinary problem of modern medicine]. Mezhdunarodnyj nauchno-issledovatelskij zhurnal [International Research Journal]. 2019;82(4-1):118-123. doi: 10.23670/ IRJ.2019.82.4.024. (Russian).

19. Chen B, Zhong L, Roush SF, Pentassuglia L, Peng X, Samaras S, Davidson J, Sawyer D, Lim C. Disruption of a GATA4/Ankrd1 signaling axis in cardiomyocytes leads to sarcomere disarray: implications for anthracycline cardiomyopathy. PLoS One. 2012;7(4):e3574. doi: 10.1371/ journal.pone.0035743.

20. Chan BYH, Roczkowsky A, Moser N, Poirier M, Hughes BG, Ilarraza R, Schulz R. Doxorubicin induces de novo expression of N-terminal-truncated matrix metalloproteinase-2 in cardiac myocytes. Canadian Journal of Physiology and Pharmacology. 2018;96(12):1238-1245. doi: 10.1139/ cjpp-2018-0275.

21. Tabish AM, Azzimato V, Alexiadis A, Buyandelger B, Knoll R. Genetic epidemiology of titin-truncating variants in the etiology of dilated cardiomyopathy. Biophysical Reviews. 2017;9(3):207-223. doi: 10.1007/s12551-0170265-7.

22. Henriksen PA. Anthracycline cardiotoxicity: an update on mechanisms, monitoring and prevention. Heart. 2018;104(12):971-977. doi: 10.1136/heartjnl-2017-3121103.

23. Fan $\mathrm{X}$, Hughes BG, Chan BYH, Launier K, Schulz R. Matrix metalloproteinase-2 in oncostatin $M-$ in- duced sarcomere degeneration in cardiomyocytes. American Journal of Physiology: Heart and Circulatory Physiology. 2016;311(1):H183-H189. doi: 10.1152/ ajpheart.00229.2016.

24. Armenian SH, Lacchetti C, Barac A, Carver J, Constine LS, Denduluri N, Dent S, Douglas PS, Durand J-B, Ewer M, Fabian C, Hudson M, Jessup M, Jones LW, Ky B, Mayer EL, Moslehi J, Oeffinger K, Ray K, Ruddy K, Daniel L. Prevention and Monitoring of Cardiac Dysfunction in Survivors of Adult Cancer: American Society of Clinical Oncology Clinical Practice Guideline. Journal of Clinical Oncology. 2017;35(8):893-911. doi: 10.1200/JCO.2016.70.5400.

25. Li S, Zhang C, Liu N, Bai H, Hou C, Song L, Pu J. Titintruncating variants are associated with heart failure events in patients with left ventricular non-compaction cardiomyopathy. Clinical Cardiology. 2019;42(5):530-535. doi: $10.1002 / \mathrm{clc} 23172$.

26. Franaszczyk M, Chmielewski P, Truszkowska G, Stawinski P, Michalak E, Rydzanicz M, SobieszczanskaMalek M, Pollak A, Szczygieł J, Kosinska J, Parulski A, Stoklosa T, Tarnowska A, Machnicki MM, Foss-Nieradko B, Szperl M, Sioma A, Kusmierczyk M, Grzybowski J, Zielinski T, Ploski R, Bilinska Z. Titin Truncating Variants in Dilated cardiomyopathy - Pprevalence and Genotype Phenotype Correlations. PLoS One. 2017;12(1):e0169007. doi: 10.1371/journal.pone.0169007.

27. Linschoten M, Teske AJ, Baas AF, Vink A, Dooijes D, Baars HF, Asselbergs FW. Truncating Titin (TTN) Variants in Chemotherapy-Induced Cardiomyopathy. Journal of Cardiac Failure. 2017;23(6):476-479. doi: 10.1016/j.cardfail.2017.03.003.

\title{
ROLE OF THE TTN, TTN-TRUNCATION, MMP-2 AND MMP-3 GENES POLYMORPHISMS IN THE DEVELOPMENT OF ANTHRACYCLINE- INDUCED CARDIOMYOPATHY
}

\author{
I. A. Karputs ${ }^{1}$, V. A. Snezhitskiy ${ }^{1}$, M. N. Kurbat ${ }^{1}$, A. Yu. Rubinskiy ${ }^{2}$, T. A. Smirnova ${ }^{3}$ \\ ${ }^{I}$ Grodno State Medical University, Grodno, Belarus \\ ${ }^{2}$ Grodno Regional Clinical Cardiology Center, Grodno, Belarus \\ ${ }^{3}$ Grodno University Clinic, Grodno, Belarus
}

\begin{abstract}
The given review is devoted to the problem of cardiotoxicity of chemotherapeutic agents. The antitumor antibiotics anthracyclines can cause cardiomyopathy, which may predict worse prognosis in cancer patients and be an independent cause of death.

Identification of patients at high risk of cardiotoxicity is the first step towards successful prevention of heart failure in cancer patients. Genetic typing is an effective measure to predict an increased risk of cardiotoxic effects of anthracyclines.

The review article analyzes the role of gene mutations in the development of chemotherapy-induced heart failure.

Keywords: chemotherapy, cardiotoxicity, protein titin, matrix metalloproteinases.

For citation: Karputs IA, Snezhitskiy VA, Kurbat MN, Rubinskij AYu, Smirnova TA. Role of polymorphisms of gene TTN, TTNtruncation, MMP-2, MMP-3 in the development of anthracycline-induced cardiomyopathy. Journal of the Grodno State Medical University. 2021;19(2):135-140. https://doi.org/10.25298/2221-8785-2021-19-2-135-140.
\end{abstract}

Конфликт интересов. Авторы заявляют об отсутствии конфликта интересов.

Conflict of interest. The authors declare no conflict of interest.

Об авторах / About the authors

*Карпуть Ирина Александровна / Karputs Iryna, e-mail: karputirina@gmail.com, ORCID: 0000-0003-0478-9419

Снежицкий Виктор Александрович / Snezhitskiy Victor, e-mail: snezh@grsmu.by, ORCID: 0000-0002-1706-1243

Курбат Михаил Николаевич / Kurbat Mikhail, e-mail: vwmisha@mail.ru, ORCID: 0000-0002-8518-2450

Рубинский Александр Юрьевич / Rubinskij Aleksandr, e-mail: kardio@mail.grodno.by

Смирнова Татьяна Анатольевна / Smirnova Tatiana, e-mail: smir-tat.anat@mail.ru

* - автор, ответственный за переписку / corresponding author 